$56^{\text {ème }}$ Congrès de la SFMBCB, 03009 (2011)

DOI: $10.1051 / \mathrm{sfmbcb} / 20115603009$

(C) Owned by the authors, published by EDP Sciences, 2011

\title{
Apports de la sédation consciente par MEOPA en Chirurgie buccale
}

\author{
Saadi-Thiers $\mathbf{K}^{\mathbf{1}}$, Fioretti $\mathbf{F}^{\mathbf{2}}$, Feki $\mathbf{A}^{\mathbf{1}}$ \\ ${ }^{1}$ UF de Médecine buccale et Chirurgie Buccale, Hôpital civil, Faculté de Chirurgie \\ dentaire, Strasbourg, France \\ ${ }^{2}$ UF d'Odontologie conservatrice et Endodontie, Hôpital civil, Faculté de Chirurgie \\ dentaire, Strasbourg, France \\ psy34@hotmail.fr
}

Les actes de chirurgie buccale sont considérés comme étant invasifs et pouvant être à l'origine d'un stress. Pour trois catégories de patients, cette anxiété peut interférer grandement avec la réalisation de la chirurgie au fauteuil et peut même la contre-indiquer : les patients présentant une anxiété importante (Hakeberg et al. 1993), ceux atteints d'un handicap psychique ou mental et ceux pour lesquels l'anxiété opératoire peut aggraver leur état pathologique (Philippart 2004).

Avec l'extension récente de l'AMM du protoxyde d'azote à l'exercice libéral, il parait d'actualité de s'interroger sur l'intérêt de son utilisation en chirurgie buccale en fonction de l'acte chirurgical à réaliser et sur sa prescription à un plus grand nombre de patients, non plus seulement en médication préventive mais aussi de confort ; en particulier avec l'essor actuel de l'implantologie.

Le MEOPA est un gaz présentant des propriétés sédatives dont l'effet est rapide et réversible (3 à 5 minutes) et qui présente peu de contre-indications. La sédation consciente obtenue correspond à un niveau de dépression minimum de la conscience qui permet au patient de maintenir une ventilation indépendante et continue, et de répondre correctement à la stimulation physique et/ou aux commandes verbales. Elle correspond au premier stade de l'analgésie selon Guedel (Guedel 1953). Il existe de nombreuses formes de sédations conscientes mais la pharmacosédation semble la plus appropriée à l'exercice dentaire (Roche 1998).

L'application de cette méthode à la chirurgie buccale augmente le confort pendant l'acte. Le patient bénéficie d'un effet analgésique supplémentaire et garde un souvenir moins traumatisant de l'acte du fait de l'effet légèrement amnésiant de la sédation. Le praticien n'est plus confronté à l'inconfort occasionné par le stress du patient et aux mouvements intempestifs associés et le risque de malaises ou de troubles du comportement est diminué. Cependant, la réalisation du geste opératoire sera modifiée par l'encombrement engendré par le matériel d'inhalation et le praticien devra adapter les différentes positions de travail en fonction de ce matériel : situation des points d'appui et de l'aspiration chirurgicale, accès visuel et instrumental. Pour certaines interventions chirurgicales dans le secteur maxillaire antérieur, cette sédation pourra alors même être contre-indiquée. En plus de l'acte opératoire, cette médication oblige le praticien à contrôler en permanence l'inhalation du gaz ainsi que le pouls et la saturation en $0_{2}$. Enfin, le praticien doit planifier une séance chirurgicale qui ne doit pas dépasser une heure.

Ainsi l'indication du MEOPA est posée en fonction de l'anxiété du patient, de sa pathologie, du type d'intervention complexe ou simple, unique ou multiple et du site opératoire. Dans certaines situations, l'anesthésie générale reste plus indiquée (Glassman 2009).

L’usage de la sédation consciente au MEOPA en chirurgie buccale présente des avantages en termes de confort et de sécurité pour le patient mais aussi en matière de santé publique par une diminution des indications de l'anesthésie générale. Par ses avantages et son extension en secteur privé, la sédation consciente pourrait s’avérer dans l'avenir d'un grand intérêt en chirurgie buccale, notamment en implantologie. 